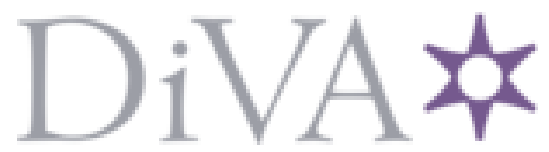

http://www.diva-portal.org

This is the published version of a paper published in IEEE Transactions on Smart Grid.

Citation for the original published paper (version of record):

Yuan, Z., Reza Hesamzadeh, M., R. Biggar, D. (2016)

Distribution Locational Marginal Pricing by Convexified ACOPF and Hierarchical Dispatch. IEEE Transactions on Smart Grid

Access to the published version may require subscription.

N.B. When citing this work, cite the original published paper.

Permanent link to this version:

http://urn.kb.se/resolve?urn=urn:nbn:se:kth:diva-203327 


\title{
Distribution Locational Marginal Pricing by Convexified ACOPF and Hierarchical Dispatch
}

\author{
Zhao Yuan, Student Member, IEEE, Mohammad Reza Hesamzadeh, Senior Member, IEEE, and Darryl R. Biggar
}

\begin{abstract}
This paper proposes a hierarchical economic dispatch (HED) mechanism for computing distribution locational marginal prices (DLMPs). The HED mechanism involves three levels: The top level is the national (regional) transmission network, the middle level is the distribution network, while the lowest level reflects local embedded networks or microgrids. Each network operator communicates its generalized bid functions (GBFs) to the next higher level of the hierarchy. The GBFs approximate the true cost function of a network by a series of affine functions. The concept of Benders cuts are employed in simulating the GBFs. The AC optimal power flow (ACOPF) is convexified and then used for dispatching generators and calculating GBFs and DLMPs. The proposed convexification is based on the second order cone reformulation. A sequential optimization algorithm is developed to tighten the proposed second order cone relaxation of ACOPF. The properties of the sequential tightness algorithm are discussed and proved. The HED is implemented in the GAMS grid computing platform. The GBFs and DLMPs are calculated for the modified IEEE 342 node low voltage test system. The numerical results show the utility of the proposed HED and GBF in implementing DLMP.
\end{abstract}

Index Terms-Convexified ACOPF, Hierarchical economic dispatch, Generalized bid function, DLMP.

\section{NOMENCLATURE}

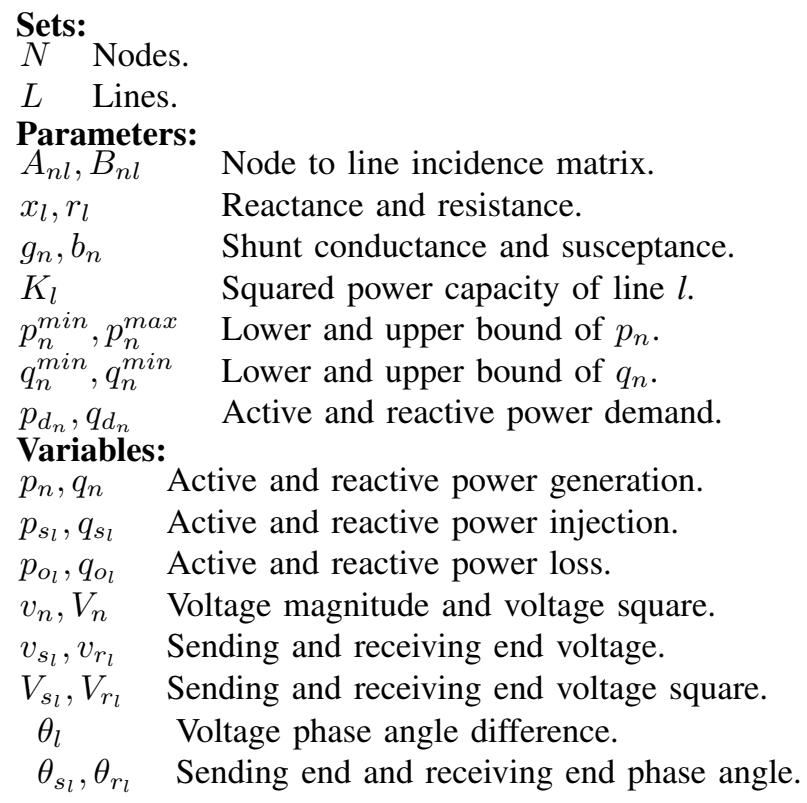

Z. Yuan and M. R. Hesamzadeh are with Electricity Market Research Group (EMReG), School of Electrical Engineering, KTH Royal Institute of Technology, Stockholm, SE-10044, Sweden. Email: yuanzhao@kth.se, mrhesamzadeh@ee.kth.se

D. R. Biggar is with the Australian Competition and Consumer Commission, Melbourne, Australia.

\section{INTRODUCTION}

$\mathbf{S}$ MART GRID advocates envisage a future in which small customers are responsive to local market conditions with devices that reduce electricity consumption at times of high prices and increase consumption at times of low prices. The increasing penetration of devices capable of responding to market prices is increasing the need for, and the utility of, improved distribution pricing signals. Efficient distribution pricing signals reflect both losses and congestion on the distribution network. Such prices vary across both time and locations and reflect the short-run marginal cost of the transportation of electricity from one point on the distribution network to another.

In recent years there has been rapidly increasing interest in locational marginal pricing of distribution networks, especially to facilitate integration of distributed energy resources [1]. The benefit of distribution locational marginal prices (DLMPs) for charging management of electric vehicles is discussed in [2]. References [3], [4] discuss boosting of demand-side responses using DLMPs. Reference [5] demonstrates how DLMPs can alleviate congestion caused by high penetration of electric vehicles (EV) and heat pumps (HP). Reference [6] proposes to integrate DLMPs and optimization in controlling future distribution networks where electronic devices are enabled to receive control signals generated from DLMP.

Given the very large number of nodes in typical distribution networks, the complexity involved in calculating DLMPs must be properly addressed. Decentralized dispatch is an attractive solution in smart grids [7]-[9]. To address the complexity, a decentralized optimal power flow (OPF) calculated by a Lagrangian-based decomposition procedure is proposed in [10]. Reference [11] reduces high-voltage radial distribution networks to simple networks by feeder reduction techniques. Reference [12] proposes the decentralized economic dispatch for smart grids using the concept of self-organizing dynamic agents. A distributed multi-agent paradigm is proposed in [13] to calculate DLMPs.

Reference [13] reports the DLMPs in a 12-bus distribution network calculated by both direct current OPF (DCOPF) and alternating current OPF (ACOPF). The results show that DLMPs from ACOPF are higher than the ones from DCOPF (this is partly because marginal loss costs are included in the ACOPF approach). The DLMP difference between DCOPF and ACOPF is larger in congestion cases (DLMP of bus 1 is $78.33 \$$ /MWh by DCOPF but $149.99 \$ / \mathrm{MWh}$ by ACOPF). On the other hand, reference [2] calculates DLMPs by DCOPF because of the ACOPF complexity in distribution networks 
with a large number of nodes.

Limitations on the ability to share network information is another issue in calculating DLMPs. The importance of coordination between the distribution and transmission network layer is discussed in [14] and [15]. This issue is significant for the operation of a power system with large-scale integration of distributed energy resources.

We can distinguish two main challenges in implementing DLMPs: (1) Computational complexity: The DCOPF assumptions are not often valid in distribution grids with high resistance to reactance $(\mathrm{R} / \mathrm{X})$ ratio [16]. On the other hand, the ACOPF in distribution networks with a large number of nodes might not be computationally tractable. This demands an OPF formulation which is accurate enough and at the same time which can be solved efficiently for distribution networks with a large number of nodes. (2) Sharing of network information: the correct calculation of DLMPs needs full information of the whole transmission, distribution and local network. Sharing of detailed network information between different network layers might not be feasible or practical. In this paper, these two challenges of implementing DLMPs are addressed.

For the computation complexity challenge, we propose a convexified ACOPF based on second order cone relaxation (SOCACOPF) [17]-[19]. The tightness of the employed relaxation is enforced by a sequential tightness algorithm. Though SemiDefinite Programming (SDP) based ACOPF ( [20]-[22]) is also one approach to solve ACOPF, the computational limits of SDP are shown in [23]. Efficient algorithms for solving SDP-based ACOPF model remains to be found [20]. SDP relaxations are exact only for limited types of problems [24][26]. Even for a 2-bus 1-generator system, SDP-based ACOPF can be infeasible and inexact [27]. [28] formulate the load flow problem of radial distribution network as conic programming by defining new variables. Our formulations do not need to define these new variables. [29] make use of polynomial optimization problem (POP) based ACOPF and then relaxed the POP model by second order cone programming. A hierarchy of SOCP problems is solved to obtain the bounds of ACOPF. The constraints in the hierarchy [29] are increasing during the solution process. On the contrast, the number of the constraints in the sequential tightness algorithm proposed in our paper does not grow in the solution process. By replacing the positive semidefinite condition with its necessary SOCP constraints, reference [30] formulate a mixed SDP/SOCP approach to improve the computational performance of the moment relaxations of ACOPF. But the numerical computation results from [30] show that the mixed SDP/SOCP are still not as fast as pure SOCP based ACOPF compared with our results. [31] use current square variables to formulate the SOCP relaxation of ACOPF and then recover voltage phase angle from solutions of other variables. In our SOC-ACOPF formulation, we explicitly include voltage phage angle variables. Thus the voltage phase angle solutions can be obtained directly.

To address the issue of sharing information between different network layers, the concept of GBF is proposed. The convexified ACOPF and the GBF are placed in a proposed HED mechanism. We prove that if the GBFs are communicated, the proposed HED achieves results very close to the global economic dispatch. We demonstrate the proposed solutions to implementing DLMP by numerical simulations using a GAMS model. The rest of this paper is organized as follows. Section II explains the convex SOC-ACOPF model and sequential tightness algorithm. Two theorems are presented in this sections to show important properties of SOC-ACOPF. Section III introduces the concept of HED mechanism and GBF. Section IV present the numerical results and discussions. The convergence of HED mechanism is proved numerically. As the communication requirement between network operators, the capacity of GBF is shown to be small. Section V concludes the advantages of the proposed SOC-ACOPF approach, HED mechanism and GBF.

\section{CONVEXIFIED ACOPF}

\section{A. Second order cone and convex envelope}

The original nonconvex ACOPF (based on branch flow model [17], [19]) is formulated in optimization problem (1).

$$
\begin{aligned}
& \underset{\Omega}{\text { Minimize }} \quad f\left(p_{n}, q_{n}, p_{o_{l}}, q_{o_{l}}\right) \\
& \text { subject to } \\
& p_{n}-p_{d_{n}}=\sum_{l}\left(A_{n l} p_{s_{l}}-B_{n l} p_{o_{l}}\right)+g_{n} V_{n}, \forall n \in N \\
& q_{n}-q_{d_{n}}=\sum_{l}\left(A_{n l} q_{s_{l}}-B_{n l} q_{o_{l}}\right)-b_{n} V_{n}, \forall n \in N \\
& p_{o_{l}}=\frac{p_{s_{l}}^{2}+q_{s_{l}}^{2}}{V_{s_{l}}} r_{l}, \forall l \in L \\
& p_{o_{l}} x_{l}=q_{o_{l}} r_{l}, \forall l \in L \\
& V_{s_{l}}-V_{r_{l}}=2 r_{l} p_{s_{l}}+2 x_{l} q_{s_{l}}-r_{l} p_{o_{l}}-x_{l} q_{o_{l}}, \forall l \in L \\
& v_{s_{l}} v_{r_{l}} \sin \theta_{l}=x_{l} p_{s_{l}}-r_{l} q_{s_{l}}, \forall l \in L \\
& p_{s_{l}}^{2}+q_{s_{l}}^{2} \leq K_{l}, \forall l \in L \\
& V_{n}=v_{n}^{2}, \forall n \in N \\
& v_{n}^{\text {min }} \leq v_{n} \leq v_{n}^{\text {max }}, \forall n \in N \\
& \theta_{l}^{\text {min }} \leq \theta_{l} \leq \theta_{l}^{\text {max }}, \forall l \in L \\
& p_{n}^{\text {min }} \leq p_{n} \leq p_{n}^{\text {max }}, \forall n \in N \\
& q_{n}^{\text {min }} \leq q_{n} \leq q_{n}^{\text {max }}, \forall n \in N
\end{aligned}
$$

Where $\Omega=\left\{p_{n}, q_{n}, p_{s_{l}}, q_{s_{l}}, p_{o_{l}}, q_{o_{l}}, V_{n}, v_{n}, \theta_{l}\right\} \in \Re$ is the set of decision variables. Equations (1b) and (1c) represent the active and reactive power balance. $A_{n l}$ and $B_{n l}$ are the incidence matrices of the network with $A_{n l}=1, B_{n l}=0$ if $n$ is sending end of branch $l$, and $A_{n l}=-1, B_{n l}=1$ if $n$ is receiving end of branch $l$. Equations (1d)-(1e) represent active power and reactive power loss. Equations (1f)-(1g) are derived by taking the magnitude and phase angle of voltage drop phasor along line $l$ respectively [17], [19]. Constraints $(1 \mathrm{j})-(1 \mathrm{~m})$ are bounds for voltage magnitude, voltage phase angle difference, active power generation and reactive power generation. Constraints (1d), (1g) and (1i) are nonconvex constraints in the optimization problem (1). Using second order cones [19], constraint (1d) can be relaxed to the rotated second order cone constraint in (2).

$$
p_{o_{l}} \geq \frac{p_{s_{l}}^{2}+q_{s_{l}}^{2}}{V_{s_{l}}} r_{l}, \forall l \in L
$$


The nonconvex constraint (1g) can be written as $z_{h_{l}}=x_{l} p_{s_{l}}-$ $r_{l} q_{s_{l}}$ where $z_{h_{l}}=v_{m_{l}} z_{\theta_{l}}, v_{m_{l}}=v_{s_{l}} v_{r_{l}}$ and $z_{\theta_{l}}=\sin \theta_{l}$. The nonconvex terms $v_{m_{l}} z_{\theta_{l}}, v_{s_{l}} v_{r_{l}}$ and $\sin \theta_{l}$ can be replaced by their convex hulls. We assume $v_{m_{l}}^{\text {min }}, z_{\theta_{l}}^{\text {min }}, v_{s_{l}}^{\text {min }}, v_{r_{l}}^{\text {min }}$ are lower bounds and $v_{m l}^{\max }, z_{\theta_{l}}^{\max }, v_{s_{l}}^{\max }, v_{r_{l}}^{\max }$ are upper bounds for the corresponding variables. The convex hull of the nonconvex terms are derived in (3) which are linear and convex. Equations (3i)-(3j) are valid for $0<\theta_{l}^{\max }<\frac{\pi}{2}$ [32]. Bounds of the variables can be determined a priori. The convex hull of $v_{s_{l}} v_{r_{l}} \sin \theta_{l}$ is expressed by three parts in (3). The first part include constraints (3a)-(3d). These constraints are the convex hull of $z_{h_{l}}=v_{m_{l}} z_{\theta_{l}}$. The second part include constraints (3e)-(3h). These constraints are the convex hull of $v_{m_{l}}=v_{s_{l}} v_{r_{l}}$. The third parts include (3i)-(3j). These constraints are the convex hull of $z_{\theta_{l}}=\sin \theta_{l}$.

$$
\begin{aligned}
& z_{h_{l}} \geqslant v_{m_{l}}^{\text {min }} z_{\theta_{l}}+z_{\theta_{l}}^{\text {min }} v_{m_{l}}-v_{m_{l}}^{\text {min }} z_{\theta_{l}}^{\text {min }}, \forall l \in L \\
& z_{h_{l}} \geqslant v_{m_{l}}^{\text {max }} z_{\theta_{l}}+z_{\theta_{l}}^{\text {max }} v_{m_{l}}-v_{m_{l}}^{\text {max }} z_{\theta_{l}}^{\text {max }}, \forall l \in L \\
& z_{h_{l}} \leqslant v_{m_{l}}^{\text {min }} z_{\theta_{l}}+z_{\theta_{l}}^{\text {max }} v_{m_{l}}-v_{m_{l}}^{\text {min }} z_{\theta_{l}}^{\text {max }}, \forall l \in L \\
& z_{h_{l}} \leqslant v_{m_{l}}^{\text {max }} z_{\theta_{l}}+z_{\theta_{l}}^{\text {min }} v_{m_{l}}-v_{m_{l}}^{\text {max }} z_{\theta_{l}}^{\text {min }}, \forall l \in L \\
& v_{m_{l}} \geqslant v_{s_{l}}^{\text {min }} v_{r_{l}}+v_{r_{l}}^{\text {min }} v_{s_{l}}-v_{s_{l}}^{\text {min }} v_{r_{l}}^{\text {min }}, \forall l \in L \\
& v_{m_{l}} \geqslant v_{s_{l}}^{\text {max }} v_{r_{l}}+v_{r_{l}}^{\text {max }} v_{s_{l}}-v_{s_{l}}^{\text {max }} v_{r_{l}}^{\text {max }}, \forall l \in L \\
& v_{m_{l}} \leqslant v_{s_{l}}^{\text {min }} v_{r_{l}}+v_{r_{l}}^{\text {max }} v_{s_{l}}-v_{s_{l}}^{\text {min }} v_{r_{l}}^{\text {max }}, \forall l \in L \\
& v_{m_{l}} \leqslant v_{s_{l}}^{\text {max }} v_{r_{l}}+v_{r_{l}}^{\text {min }} v_{s_{l}}-v_{s_{l}}^{\text {max }} v_{r_{l}}^{\text {min }}, \forall l \in L \\
& z_{\theta_{l}} \geqslant \cos \left(\frac{\theta_{l}^{\text {max }}}{2}\right)\left(\theta_{l}+\frac{\theta_{l}^{\text {max }}}{2}\right)-\sin \left(\frac{\theta_{l}^{\text {max }}}{2}\right), \forall l \in L \\
& z_{\theta_{l}} \leqslant \cos \left(\frac{\theta_{l}^{\text {max }}}{2}\right)\left(\theta_{l}-\frac{\theta_{l}^{\text {max }}}{2}\right)+\sin \left(\frac{\theta_{l}^{\text {max }}}{2}\right), \forall l \in L
\end{aligned}
$$

In a similar way the convex hull of constraint (1i) is derived in (4).

$$
\begin{aligned}
& V_{n} \geq v_{n}^{2}, \forall n \in N \\
& V_{n} \leq\left(v_{n}^{\text {max }}+v_{n}^{\text {min }}\right) v_{n}-v_{n}^{\text {max }} v_{n}^{\text {min }}, \forall n \in N
\end{aligned}
$$

Using (2), (3) and (4), the optimization problem (1) can now be reformulated as a second order cone (SOC) program. This proposed SOC-ACOPF represented by [(1a)-(1c),(1e)(1f),(1j)-(1m)] can be solved efficiently using interior point method (IPM) [33].

\section{B. Sequential tightness algorithm}

The conic relaxations in (2) are not guaranteed to be tight. To deal with this problem, we propose a sequential algorithm to improve the tightness. This sequential algorithm is based on Theorem 1 and Theorem 2 below:

\section{Theorem 1}

Assume that:

(1). the objective function of nonconvex ACOPF (1) and the proposed SOC-ACOPF is convex;

(2). nonconvex ACOPF (1) has exactly one global optimal solution $\left(p_{s_{l}}^{*}, q_{s_{l}}^{*}, p_{o_{l}}^{*}\right)$;

(3). non-exact optimal solution (if exists) of SOC-ACOPF $p_{o_{l}}^{\prime}>p_{o_{l}}^{*}$.

Then, constraint $p_{o_{l}} \leq p_{o_{l}}^{*}$ guarantees the tightness of constraint (2).
Proof. Firstly, we consider the case that SOC-ACOPF is exact (the relaxations in (2)-(4) are tight). The proof of theorem 1 is based on theorem 3 in reference [18] which proves that second order conic optimal power flow (SOPF) has at most one optimal solution when SOPF is exact for a radial network. Because our SOC-ACOPF model has one more constraint (1g) (to make our model valid for both radial and meshed networks) than the SOPF model described in reference [18], we are actually reducing the feasible region of SOPF in reference [18]. This means that either we keep the unique optimal solution in the feasible region or we exclude the optimal solution. For both cases, the conclusion that there is at most one global optimal solution of SOC-ACOPF when SOC-ACOPF is exact is still valid. Assume the exact optimal solution of SOC-ACOPF is $p_{o_{l}}^{e}$. If $p_{o_{l}}^{e} \neq p_{o_{l}}^{*}$, then $p_{o_{l}}^{e}$ must be the global optimal solution of the nonconvex ACOPF since $f^{e} \leq f^{*}$. Where $f^{e}$ and $f^{*}$ are the value of objective function for SOC-ACOPF and the nonconvex ACOPF respectively. This contradicts our assumption that there is exactly one global optimal solution of the nonconvex ACOPF. So $p_{o_{l}}^{e} \neq p_{o_{l}}^{*}$ is not valid. Constraint $p_{o_{l}} \leq p_{o_{l}}^{*}$ is feasible for SOC-ACOPF when it is exact.

When SOC-ACOPF is not exact, assume $\left(p_{s_{l}}^{\prime}, q_{s_{l}}^{\prime}, p_{o_{l}}^{\prime}, V_{s_{l}}^{\prime}\right)$ is the optimal solution of SOC-ACOPF after we have put the constraint $p_{o_{l}} \leq p_{o_{l}}^{*}$ :

$$
p_{o_{l}}^{*} \geq p_{o_{l}}^{\prime} \geq \frac{p_{s_{l}}^{\prime 2}+q_{s_{l}}^{\prime 2}}{V_{s_{l}}^{\prime}} r_{l}
$$

If $p_{o_{l}}^{\prime} \neq \frac{p_{s_{l}}^{\prime 2}+q_{s_{l}}^{\prime 2}}{V_{s_{l}}^{\prime}} r_{l}$, then:

$$
p_{o_{l}}^{\prime}>p_{o_{l}}^{*}
$$

(6) contradicts (5). So $p_{o_{l}}^{\prime} \neq \frac{p_{s_{l}}^{\prime 2}+q_{s_{l}}^{\prime 2}}{V_{s_{l}}^{\prime}} r_{l}$ is not valid. Constraint (2) must be tight.

Theorem 1 shows that as long as we have a good estimation of $p_{O_{l}}^{*}$, then we can tighten constraint (2). The problem is that it is difficult to estimate $p_{o_{l}}^{*}$ before solving nonconvex ACOPF. We propose the following theorem to design the tightness algorithm.

\section{Theorem 2}

If the assumptions in theorem 1 hold and the decreasing sequence $p_{o_{l}, i} \leq p_{o_{l}, i-1}$ converges to $p_{o_{l}}^{*}$ which is the optimal solution of nonconvex ACOPF, then sequential constraints $p_{o_{l}} \leq p_{o_{l}, i}$ guarantee the tightness of constraint (2).

Proof. The proof of theorem 2 is based on theorem 1 and L'Hopital's rule. We denote here the sequential optimization problem constrained by $p_{o_{l}} \leq p_{o_{l}, i}$ as SOC-ACOPF- $i$ where $i$ is the index for the sequence. Because SOC-ACOPF is assumed to be feasible for $p_{o_{l}} \leq p_{o_{l}}^{*}$ and $p_{o_{l}, i} \geq p_{o_{l}}^{*}$, SOC$\mathrm{ACOPF}-i$ is always feasible. If the solution of SOC-ACOPF- $i$ is denoted as $\left(p_{s_{l}, i}^{*}, q_{s_{l}, i}^{*}, p_{o_{l}, i}^{*}, V_{s_{l}, i}^{*}\right)$, we have:

$$
p_{o_{l}, i} \geq p_{o_{l}, i}^{*} \geq \frac{p_{s_{l}, i}^{* 2}+q_{s_{l}, i}^{* 2}}{V_{s_{l}, i}^{*}} r_{l}
$$


From theorem 1, $p_{o_{l}} \leq p_{o_{l}}^{*}$ guarantees the tightness of equation (2):

$$
\lim _{i \rightarrow+\infty} \frac{p_{s_{l}, i}^{* 2}+q_{s_{l}, i}^{* 2}}{V_{s_{l}, i}^{*}} r_{l}=p_{o_{l}}^{*}
$$

And we have assumed in theorem 2 that sequence $p_{o_{l}, i} \leq$ $p_{o_{l}, i-1}$ converges to $p_{o_{l}}^{*}$ :

$$
\lim _{i \rightarrow+\infty} p_{o_{l}, i}=p_{o_{l}}^{*}
$$

According to L'Hopital's rule, (7)-(9) imply:

$$
\lim _{i \rightarrow+\infty} p_{o_{l}, i}^{*}=p_{o_{l}}^{*}
$$

In other words, the solutions of sequential optimization SOCACOPF- $i$ converge to where equation (2) is tight.

Theorem 2 suggests we can find optimal solution of SOCACOPF by solving sequential SOC-ACOPF- $i$. The challenge is how to design or calculate the proper sequence $p_{o_{l}, i}$ converging to $p_{o_{l}, i}^{*}$. Here we propose to begin with a rather rough estimation of $p_{o_{l}}^{*}$ and then iteratively improve the estimation quality. The numerical results show that the proposed tightness algorithm can make the relaxation in constraint (2) tight.

Given (1h), the initial estimation $K_{o_{l}, 0}$ is simply calculated by (11):

$$
K_{o_{l}, 0}=\frac{K_{l}}{V_{s_{l}}^{\min }} r_{l}
$$

Where $V_{s_{l}}^{\min }$ is the lower bound of $V_{s_{l}}$. The sequential SOCACOPF- $i$ is then constrained by (12):

$$
p_{o_{l}} \leq K_{o_{l}, i}, \forall l \in L
$$

Where $K_{o_{l}, i}, i \in\{0,1,2, \ldots, M\}$ is the $i^{t h}$ estimation of $p_{o_{l}}^{*}$. The tightness algorithm works by iteratively reducing the upper bounds $K_{o_{l}, i}$ of power loss constraints that violate tightness criterion $\varepsilon$. If $p_{o_{l}}-\frac{p_{s_{l}}^{2}+q_{s_{l}}^{2}}{V_{s_{l}}} r_{l}>\varepsilon, K_{o_{l}, i+1}=\alpha_{l} K_{o_{l}, i}$, where $0<\alpha_{l}<1$ is the decreasing parameter. The proposed sequential tightness algorithm is summarized in algorithm 1. This algorithm is illustrated in Fig. 1 where $X^{*}$ denotes the

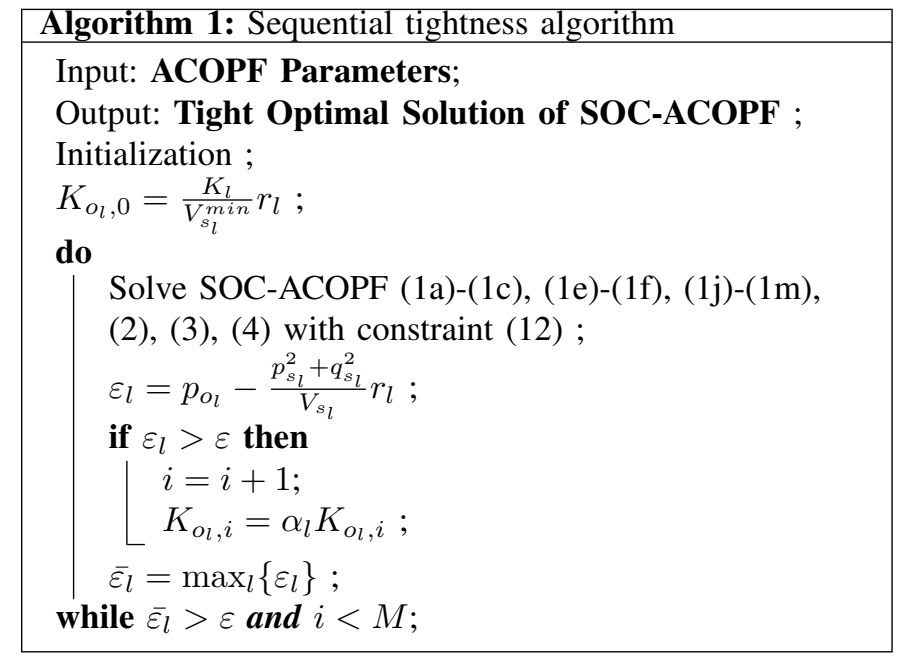

final solution. $M$ denotes the maximum number of iterations specified a priori. The original nonconvex feasible region of ACOPF is plotted with a solid line in Fig. 1. The convex feasible region of proposed SOC-ACOPF is plotted with a dashed line in Fig. 1. We will show the performance of this algorithm in Section 3 of this paper. It is worth to mention that the proposed sequential tightness algorithm is only tight for the relaxation in (2). Because there are additional relaxations we have introduced in (3a)-(4b), the final solutions of the proposed SOC-ACOPF model are generally not tight for the constraint (1g) in ACOPF. Using interior point method to solve the proposed SOC-ACOPF model in polynomial time does not violate the NP-hardness proof [34], [35] of ACOPF because the proposed SOC-ACOPF is still a relaxed model of ACOPF.

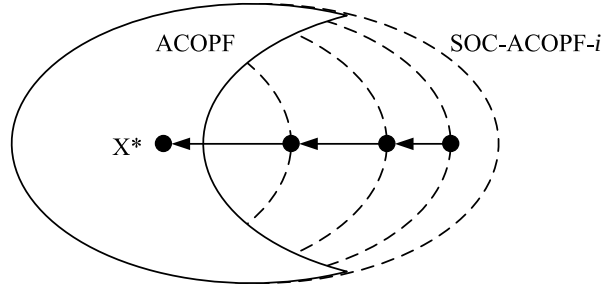

Fig. 1. The conceptual diagram of the proposed sequential tightness algorithm

\section{THE HIERARCHICAL ECONOMIC DISPATCH}

To define hierarchical economic dispatch (HED), we should firstly explain the traditional centralized economic dispatch (CED). We assume here centralized economic dispatch as one system operator dispatching all generations in transmission, distribution and local networks. To fulfill this dispatch task, the system operator should obtain all network information about his responsible area. Mathematically, CED is to solve ACOPF problem (1) or SOC-ACOPF [(1a)-(1c),(1e)-(1f),(1j)$(1 \mathrm{~m})]$. The CED is a very large scale optimization problem considering the enormous nodes, lines and DERs. To release the complexity of CED, we propose the HED mechanism in this paper. HED actually decompose CED by Benders decomposition. We show that Benders cuts in this proposed hierarchical economic dispatch have specific economic meanings in the defined GBF.

\section{A. The hierarchical economic dispatch mechanism}

We propose a three-level dispatch mechanism with each network operator responsible for its own network. The network layers are connected through tie lines. At the third level, local network or microgrid operators (LNOs) carry out their own dispatch considering local network constraints. LNOs communicate dispatch results through the proposed GBFs to the second level of hierarchy. At the second level, the distribution network operators (DNOs) run another optimization problem taking into account the submitted GBFs from all connected LNOs and the second-level network constraints. The results of the second level optimization is packed in the form of GBFs and submitted to the first level of hierarchy. At the first level of hierarchy, transmission network operator (TNO) solves the dispatch problem of transmission network taking into account 
the submitted GBFs from DNOs. Once the top level of the hierarchy is completed, the dispatch results are determined and the resulting nodal prices can be computed and communicated back down to the hierarchy. This is illustrated in Fig. 2.

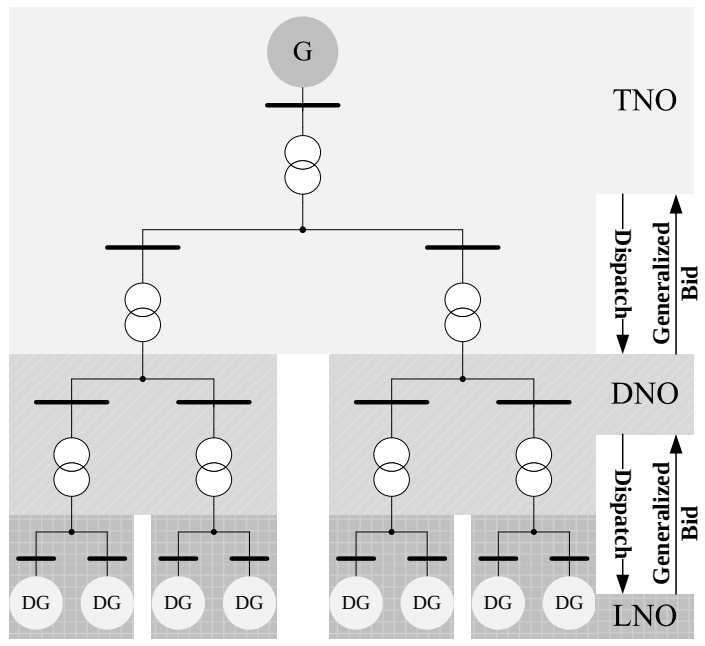

Fig. 2. The conceptual diagram of the proposed HED mechanism

\section{B. Generalized bid function}

The concept of GBF is proposed to avoid communicating detailed network and regional bid information between dispatch levels. The HED problem for LNOs contingent on the total power generation is set out in (13).

$$
\begin{aligned}
F_{k}\left(P_{k, j}^{\text {sum }}\right)= & \text { Minimize } \\
& \sum_{n \in N_{k}} f\left(p_{n}, q_{n}\right) \\
\text { subject to } & (1 b),(1 c),(1 e),(1 f),(1 j)-(1 m) \\
& (2),(3),(4),(12) \\
& \sum_{n \in N_{k}} p_{n}=P_{k, j}^{\text {sum }}:\left(\alpha_{k, j}\right)
\end{aligned}
$$

Where $F_{k}$ is the cost of local network dispatch as a function of its total power generation $P_{k, j}^{s u m} . k \in K$ is the index of the LNO. $f\left(p_{n}, q_{n}\right)$ is the generation cost of the local DGs. $N_{k}$ is the set of local nodes. $j \in J_{k}$ is the index of GBFs. If we use $S\left(P_{k, j}^{s u m}\right)$ to denote the feasible region of $\left(p_{n}, q_{n}\right)$ as a function of $P_{k, j}^{s u m}$. The optimization value function $F_{k}\left(P_{k, j}^{\text {sum }}\right)$ is convex because: (1). $f\left(p_{n}, q_{n}\right)$ is jointly convex on $\left\{\left(p_{n}, q_{n}, P_{k, j}^{\text {sum }}\right) \mid\left(p_{n}, q_{n}\right) \in S\left(P_{k, j}^{\text {sum }}\right), P_{k, j}^{\text {sum }} \in \Re^{+}\right\}$; (2). $S\left(P_{k, j}^{\text {sum }}\right)$ is convex on $\Re^{+}$; and (3). $\Re^{+}$is convex (see proposition 2.1 in reference [36]). $F_{k}$ can be approximated from below by a set of affine functions as in (14) shown by Fig. 3.

$$
\begin{gathered}
F_{k}\left(P_{k, j}^{s u m}\right) \geq \hat{F}_{k, j}+\hat{\alpha}_{k, j}\left(\sum_{n \in N_{k}} p_{n}-\hat{P}_{k, j}^{s u m}\right) \\
\forall j \in J_{k}, \forall k \in K
\end{gathered}
$$

We define GBF as the set of parameters of the affine approximator (14). These parameters are communicated through set $L N O_{k}=\left\{\left(\hat{F}_{k, j}, \hat{\alpha}_{k, j}, \hat{P}_{k, j}^{s u m}\right): j \in J_{k}\right\}$ to the DNO. Once all

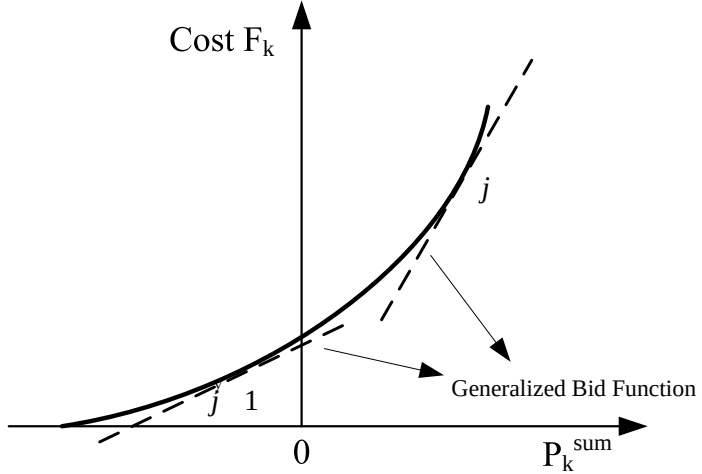

Fig. 3. Approximation of a convex cost function by affine functions

$L N O_{k}$ sets are communicated, the DNO solves the following dispatch problem (15).

$$
\begin{aligned}
G_{v}\left(P_{v, j}^{\text {sum }}\right)=\text { Minimize } & \sum_{n \in N_{v}} f\left(p_{n}, q_{n}\right)+\sum_{k \in K} F_{k} \\
\text { subject to } & (1 b),(1 c),(1 e),(1 f),(1 j)-(1 m) \\
& (2),(3),(4),(12),(14) \\
& \sum_{n \in N_{v}} p_{n}=P_{v, j}^{\text {sum }}:\left(\alpha_{v, j}\right)
\end{aligned}
$$

Same as $F_{k}, G_{v}$ is also a convex optimization value function based on proposition 2.1 in reference [36]. $G_{v}$ can be approximated from below by affine functions (16b). $v \in V$ is the index of the DNO. The parameters of these affine functions are communicated through set $D N O_{v}=\left\{\left(\hat{G}_{v, j}, \hat{\alpha}_{v, j}, \hat{P}_{v, j}^{\text {sum }}\right)\right.$ : $\left.j \in J_{v}\right\}$ to TNO. TNO solves the optimization problem (16).

$$
\begin{aligned}
& \text { Minimize } \sum_{n \in N_{t}} f_{t}\left(p_{n}, q_{n}\right)+\sum_{v \in V} G_{v} \\
& \text { subject to }(1 b),(1 c),(1 e),(1 f),(1 j)-(1 m) \\
&(2),(3),(4),(12) \\
& G_{v}\left(P_{v, j}^{\text {sum }}\right) \geq \hat{G}_{v, j}+\hat{\alpha}_{v, j}\left(\sum_{n \in N_{v}} p_{n}-P_{v, j}^{\text {sum }}\right) \\
& \forall j \in J_{v}, \forall v \in V
\end{aligned}
$$

Once optimization problem (16) is solved, TNO finds the nodal prices. It also communicates the total power generation requirement $\hat{P}_{v, \hat{j}}^{\text {sum }}$ to DNOs $(\hat{j}$ is the index of final GBF for which the HED converges). Given $\hat{P}_{v, \hat{j}}^{s u m}$, DNOs find the nodal prices for their networks. The DNO also communicates $\hat{P}_{k, \hat{j}}^{s u m}$ to the connected LNOs. LNOs then calculate the nodal prices for their local networks. The underlying mathematical structure behind the HED mechanism is the Benders decomposition approach. The proposed HED relaxes the decomposition of economic dispatch by taking $P_{k(v), j}^{s u m}$ as the complicating variables instead of the tie-line power flows $p_{s l}, q_{s l}$. This leads to fewer GBFs when multiple tie-lines exist. In this way, DNOs or LNOs do not need to submit GBFs for each tie-line ( $F_{k}$ and $G_{v}$ are not functions of tie-line power flows). Constraints (14) and (16b) are actually Benders cuts for aggregated tie lines. If the problem is convex, it is proved that optimal solution can be found within finite iterations [37]. 


\begin{tabular}{|l|}
\hline Algorithm 2: Hierarchical Economic Dispatch Mechanism \\
\hline Initialization; \\
TNO solve transmission network dispatch (16); \\
TNO broadcast $\hat{P}_{v, \hat{j}}^{s u m}$ to DNOs; \\
DNOs solve distribution network dispatch (15); \\
DNOs broadcast $\hat{P}_{v, \hat{j}}^{s u m}$ to LNOs; \\
DNOs submit $D N O_{k}$ to TNO; \\
LNOs solve local network dispatch (14); \\
LNOs submit $L N O_{k}$ to DNO; \\
\hline
\end{tabular}

\section{NUMERICAL RESUlTS AND DISCUSSION}

The proposed SOC-ACOPF and its tightness algorithm are examined through several case studies. Then the proposed HED is simulated. All the models are coded in GAMS. MATPOWER and LINDOGLOBAL are used for comparison. Simulations are run on a PC with Intel i7-2760QM 2.4 GHz CPU and 8 GB of RAM.

\section{A. The performance of proposed SOC-ACOPF}

The results of SOC-ACOPF with sequential tightness algorithm are reported in Table I and Table II. The relaxation gap of constraints (2) is calculated as:

$$
G p_{o_{l}}=\max _{l}\left\{p_{o_{l}}-\frac{p_{s_{l}}^{2}+q_{s_{l}}^{2}}{V_{s_{l}}} r_{l}\right\}
$$

The stopping criteria for the SOC-ACOPF iteration is $G p_{o_{l}}<$ $10^{-9}$. In all reported cases, the $G p_{O_{l}}$ indicator is less than $10^{-9}$. The optimality gap of the proposed SOC-ACOPF is measured in $G p_{f}=\frac{f^{*}-f}{f^{*}} \times 100 \%$. In the calculation of $G p_{f}$, $f^{*}$ is the global solution of nonconvex ACOPF calculated by LINDOGLOBAL and $f$ is the solution of proposed SOCACOPF found by MOSEK solver.

TABLE I

THE PERFORMANCE OF SEQUENTIAL TIGHTNESS ALGORITHM: OBJECTIVE SOLUTION

\begin{tabular}{|c|c|c|c|c|c|}
\hline \multirow{2}{*}{ Case } & \multicolumn{3}{|c|}{ Objective [\$] } & \multirow{2}{*}{$G p_{o_{l}}$} & \multirow{2}{*}{$G p_{f}[\%]$} \\
\cline { 2 - 4 } & SOC & matpower & Global & & \\
\hline IEEE14 & 8076.99 & 8081.53 & 8078.80 & 0 & 0.02 \\
IEEE57 & 41673.08 & 41737.79 & 41698.64 & 0 & 0.06 \\
IEEE118 & 129619.60 & 129660.70 & 129626.45 & 0 & 0.01 \\
IEEE300 & 718109.18 & 719725.11 & 719459.62 & 0 & 0.18 \\
\hline
\end{tabular}

TABLE II

THE PERFORMANCE OF SEQUENTIAL TIGHTNESS ALGORITHM: COMPUTATION TIME

\begin{tabular}{|c|c|c|c|}
\hline \multirow{2}{*}{ Case } & \multicolumn{3}{|c|}{ CPU time [s] } \\
\cline { 2 - 4 } & SOC & matpower & Global \\
\hline IEEE14 & 0.10 & 0.11 & 0.20 \\
IEEE57 & 0.19 & 0.12 & 2.31 \\
IEEE118 & 0.33 & 0.30 & 27.1 \\
IEEE300 & 3.10 & 0.40 & 257.68 \\
\hline
\end{tabular}

As we can see from Table I, the results from SOCACOPF is very close to the global solutions obtained by LINDOGLOBAL.

\section{B. Convergence of hierarchical economic dispatch}

The IEEE 342 node network [38] is modified here to illustrate the operation of the proposed HED. This test case has transmission network (nodes P1-P4, P7-P8), distribution network (nodes P5-P6, P9-P390) and local networks (nodes S193-S240 in the eight spot $277 / 480 \mathrm{~V}$ networks). The local networks are connected to the distribution network by transformers denoted as X3, X4 to X22 in Fig. 6. One $50 \mathrm{MW}$ generator is located in the transmission network. Each $13.2 \mathrm{kV}$ distribution feeder is equipped with one 7.5 MW distributed generator (DG). We distribute 48 DGs among all nodes of the local networks (one 3 MW DG at each node). To simulate congestion in the distribution network and local networks, we increase the load levels in all the local networks by four times and then reduce the tieline transformer capacity (X10-X18 is reduced to 3 MVA and $\mathrm{X} 21-\mathrm{X} 22$ is reduced to $4 \mathrm{MVA}$ ). We assume all generators are dispatchable. To accelerate computation, we design a GAMS grid computing structure to assign the dispatch task of each network to different threads as demonstrated in Fig. 5.

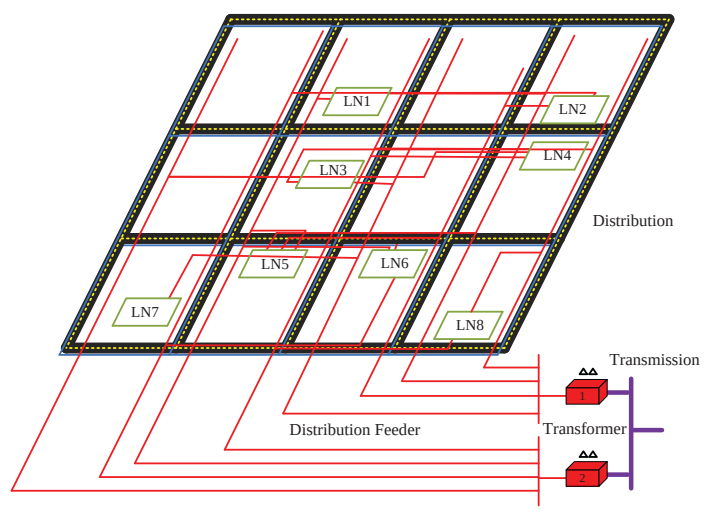

Fig. 4. The hierarchy of the modified IEEE 342 node test system

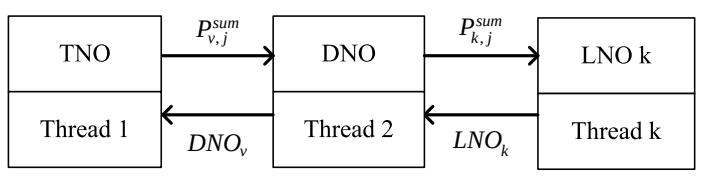

Fig. 5. The GAMS grid computing structure of hierarchical economic dispatch

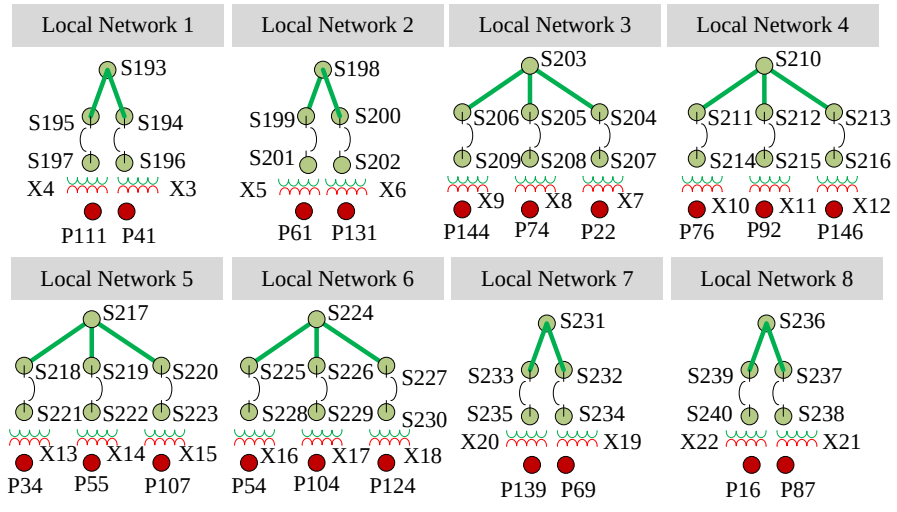

Fig. 6. Local low voltage networks 
The dispatch results of HED are listed in Table III. We denote the case of no congestion as the base case in this paper. CED results denoted as ' $\mathrm{C}$ ' are also listed. The HED results (denoted as ' $\mathrm{H}$ ') converge to the solution very close to centralized dispatch dispatch cost. All cases converge within three iterations shown in Fig. 7. Because of approximations used in HED mechanism, the final cost of HED is a bit different from centralized dispatch (the difference is within $1 \%$ after three iterations). The CPU time for computation is within 1.8 second. If the LNOs and DNO submit their GBFs (i.e. parameters of three affine approximators in one package) to the next higher hierarchy, the HED converges in one iteration. It is worth to mention that Benders decomposition is a way to build GBFs in HED. HED does not work in an iterative way.

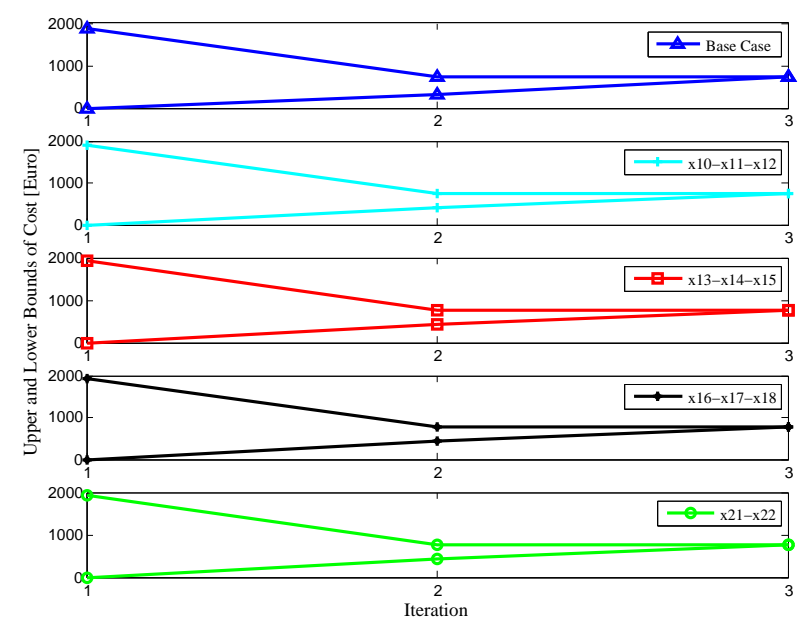

Fig. 7. The convergence of the proposed HED mechanism

\section{Distribution locational marginal price}

The nodal prices are shown in Fig. 8. All nodal prices are very close to each other in the base case. The only differences in the nodal prices are due to the marginal cost of energy loss. This can be clearly observed from the small price spikes at the ending nodes (S193, S198, S203, S210, S217, S224, S231 and S236) of each local network. The congested distribution transformers are indicated in the legend of Fig. 8. When congestion happens, the nodes located in the local network have higher prices. The consumers with priceresponsive load can response to these higher prices. This can be observed by comparing consumer payment and DG income when congestion happens with the ones in the base case. We plot the total payment of consumers in the local networks in Fig. 9. The payment difference as compared to the base-case payments is also shown in Fig. 9. The congestion management potential of DLMP can be further demonstrated by the income increase of DGs when congestion happens. This is shown in Fig. 10. When congestion happens, the increased nodal prices give local DGs strong incentive to produce more energy.

\section{The generalized bid function}

The GBFs are listed in Tables IV-VIII. We sum the $\hat{F}_{k, j}$ and $\hat{G}_{v, j}$ parameters in the GBFs for all networks and list the

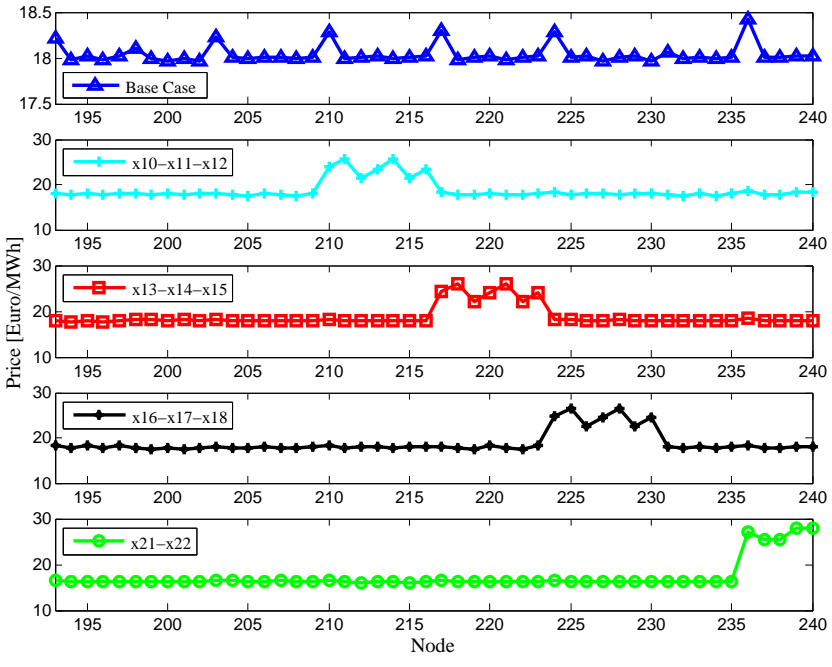

Fig. 8. The nodal prices in local networks

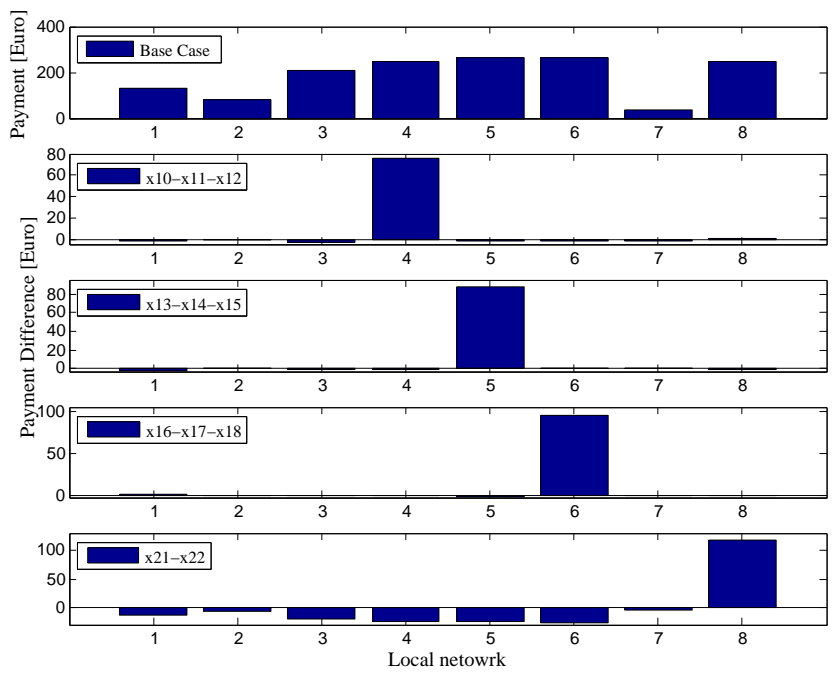

Fig. 9. The total payment of consumers in local networks
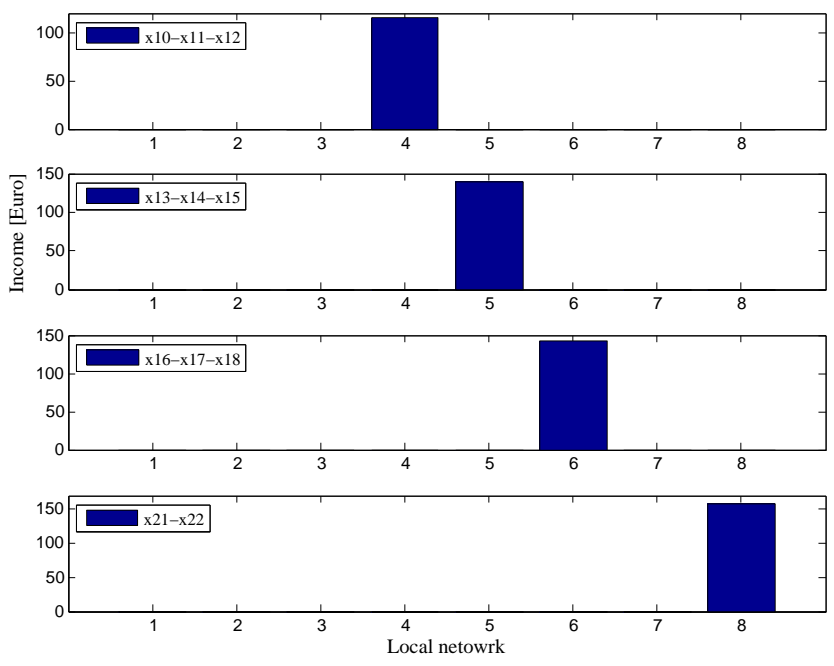

Fig. 10. The total income of DGs in local networks 
TABLE III

THE RESUlts OF THE ACTIVE POWER DISPATCH FROM HED MECHANISM, C: CENTRALIZED DISPATCH, H: HED

\begin{tabular}{|c|c|c|c|c|c|c|c|c|c|c|c|}
\hline \multirow{2}{*}{ Network } & \multirow{2}{*}{ Generator } & \multicolumn{2}{|c|}{ Base Case [MW] } & \multicolumn{2}{|c|}{ x10-x11-x12 [MW] } & \multicolumn{2}{|c|}{ x13-x14-x15 [MW] } & \multicolumn{2}{|c|}{ x16-x17-x18 [MW] } & \multicolumn{2}{|c|}{ x21-x22 [MW] } \\
\hline & & $\mathrm{C}$ & $\mathrm{H}$ & $\mathrm{C}$ & $\mathrm{H}$ & $\mathrm{C}$ & $\mathrm{H}$ & $\mathrm{C}$ & $\mathrm{H}$ & $\mathrm{C}$ & $\mathrm{H}$ \\
\hline Transmission & 1 & 50.00 & 50.00 & 50.00 & 50.00 & 50.00 & 50.00 & 50.00 & 50.00 & 50.00 & 50.00 \\
\hline \multirow{8}{*}{ Distribution } & 2 & 7.50 & 7.50 & 7.50 & 7.50 & 7.50 & 7.50 & 7.50 & 7.50 & 7.50 & 7.50 \\
\hline & 3 & 7.50 & 7.50 & 7.50 & 7.50 & 7.50 & 7.50 & 7.50 & 7.50 & 7.50 & 7.50 \\
\hline & 4 & 7.50 & 7.50 & 7.50 & 7.50 & 7.50 & 7.50 & 7.50 & 7.50 & 7.50 & 7.50 \\
\hline & 5 & 7.50 & 7.50 & 7.50 & 7.50 & 7.50 & 7.50 & 7.50 & 7.50 & 7.50 & 7.50 \\
\hline & 6 & 7.50 & 7.50 & 7.50 & 7.50 & 7.50 & 7.50 & 7.50 & 7.50 & 7.50 & 7.50 \\
\hline & 7 & 7.50 & 7.50 & 7.50 & 7.50 & 7.50 & 7.50 & 7.50 & 7.50 & 7.50 & 7.50 \\
\hline & 8 & 7.50 & 7.50 & 7.50 & 7.50 & 7.50 & 7.50 & 7.50 & 7.50 & 7.50 & 7.50 \\
\hline & 9 & 5.93 & 5.96 & 0.99 & 0.94 & 0.14 & 0.15 & 0.10 & 0.10 & 0.05 & 0.04 \\
\hline \multirow{2}{*}{ Local 4} & 29 & 0.00 & 0.00 & 2.89 & 3.00 & 0.00 & 0.00 & 0.00 & 0.00 & 0.00 & 0.00 \\
\hline & 30 & 0.00 & 0.00 & 1.40 & 2.07 & 0.00 & 0.00 & 0.00 & 0.00 & 0.00 & 0.00 \\
\hline \multirow{3}{*}{ Local 5} & 36 & 0.00 & 0.00 & 0.00 & 0.00 & 2.44 & 3.00 & 0.00 & 0.00 & 0.00 & 0.00 \\
\hline & 37 & 0.00 & 0.00 & 0.00 & 0.00 & 0.17 & 2.86 & 0.00 & 0.00 & 0.00 & 0.00 \\
\hline & 38 & 0.00 & 0.00 & 0.00 & 0.00 & 0.18 & 0.00 & 0.00 & 0.00 & 0.00 & 0.00 \\
\hline \multirow{3}{*}{ Local 6} & 43 & 0.00 & 0.00 & 0.00 & 0.00 & 0.00 & 0.00 & 2.44 & 3.00 & 0.00 & 0.00 \\
\hline & 44 & 0.00 & 0.00 & 0.00 & 0.00 & 0.00 & 0.00 & 2.87 & 2.97 & 0.00 & 0.00 \\
\hline & 45 & 0.00 & 0.00 & 0.00 & 0.00 & 0.00 & 0.00 & 0.65 & 0.00 & 0.00 & 0.00 \\
\hline \multirow{3}{*}{ Local 8} & 53 & 0.00 & 0.00 & 0.00 & 0.00 & 0.00 & 0.00 & 0.00 & 0.00 & 2.98 & 3.00 \\
\hline & 54 & 0.00 & 0.00 & 0.00 & 0.00 & 0.00 & 0.00 & 0.00 & 0.00 & 3.00 & 3.00 \\
\hline & 55 & 0.00 & 0.00 & 0.00 & 0.00 & 0.00 & 0.00 & 0.00 & 0.00 & 0.00 & 0.13 \\
\hline \multicolumn{2}{|c|}{ Total Cost $[€]$} & 731.67 & 732.25 & 757.91 & 755.09 & 766.93 & 762.38 & 769.15 & 766.96 & 769.15 & 776.38 \\
\hline
\end{tabular}

accumulated results in the last row of the Tables IV-VIII. As in Tables III-VII, the LNOs submit their calculated GBFs to the DNO. Accordingly, the DNO takes into account submitted GBFs from LNOs and prepars its GBFs to be submitted to the TNO. The TNO calculates the dispatch instructions and nodal prices will be communicated back down to the hierarchy. Note that LNOs only need to submit their GBFs to the DNO. Bids from the 48 local DGs are not required to be submitted to the DNO. In other words, with GBFs, the network layers would not need to share their detailed network information between each others. Table IV - Table VIII demonstrate the communication burden of HED is small.

TABLE IV

THE GBFs IN BASE CASE

\begin{tabular}{|c|c|c|c|c|c|c|}
\hline \multirow{2}{*}{ Network } & \multicolumn{2}{|c|}{$j=1$} & \multicolumn{2}{c|}{$j=2$} & \multicolumn{2}{c|}{$j=3$} \\
\cline { 2 - 7 } & $P^{\text {sum }}$ & $\alpha$ & $P^{\text {sum }}$ & $\alpha$ & $P^{\text {sum }}$ & $\alpha$ \\
\hline DNO & 21.60 & 8.00 & 58.46 & 18.00 & 58.46 & 18.00 \\
LNO1 & 7.20 & 24.00 & 0.00 & 20.00 & 0.00 & 19.98 \\
LNO2 & 6.64 & 24.50 & 0.00 & 20.50 & 0.00 & 20.48 \\
LNO3 & 10.17 & 26.50 & 0.00 & 21.00 & 0.00 & 20.98 \\
LNO4 & 10.67 & 27.00 & 0.00 & 21.50 & 0.00 & 21.47 \\
LNO5 & 10.86 & 27.50 & 0.00 & 22.00 & 0.00 & 21.98 \\
LNO6 & 10.88 & 28.00 & 0.00 & 22.50 & 0.00 & 22.48 \\
LNO7 & 6.25 & 27.00 & 0.00 & 23.00 & 0.00 & 22.95 \\
LNO8 & 8.37 & 27.50 & 0.00 & 23.50 & 0.00 & 23.45 \\
\hline Cost [€] & 1825.28 & 632.25 & \multicolumn{2}{c|}{632.25} \\
\hline
\end{tabular}

\section{CONClusion}

This paper proposes solutions to practical challenges with implementing locational marginal prices in distribution networks. The challenges considered here include (1) the computational complexity of nodal prices in AC optimal power flow and (2) the network information which potentially must be communicated. We propose a SOC-ACOPF to calculate nodal prices in a distribution network. The proposed SOC-ACOPF can be solved efficiently to global optimality while it has more
TABLE V

THE GBFS IN CASE OF X10-X11-X12 CONGESTION

\begin{tabular}{|c|c|c|c|c|c|c|}
\hline \multirow{2}{*}{ Network } & \multicolumn{2}{|c|}{$j=1$} & \multicolumn{2}{c|}{$j=2$} & \multicolumn{2}{c|}{$j=3$} \\
\cline { 2 - 7 } & $P^{\text {sum }}$ & $\alpha$ & $P^{\text {sum }}$ & $\alpha$ & $P^{\text {sum }}$ & $\alpha$ \\
\hline DNO & 20.32 & 8.00 & 53.44 & 18.00 & 53.44 & 18.00 \\
LNO1 & 7.10 & 24.00 & 0.00 & 19.98 & 0.00 & 20.00 \\
LNO2 & 6.55 & 24.50 & 0.00 & 20.48 & 0.00 & 20.49 \\
LNO3 & 10.00 & 26.50 & 0.00 & 20.98 & 0.00 & 20.99 \\
LNO4 & 13.77 & 27.50 & 5.07 & 23.50 & 5.07 & 23.50 \\
LNO5 & 10.69 & 27.50 & 0.00 & 21.98 & 0.00 & 21.99 \\
LNO6 & 10.71 & 28.00 & 0.00 & 22.48 & 0.00 & 22.49 \\
LNO7 & 6.16 & 27.00 & 0.00 & 22.98 & 0.00 & 22.98 \\
LNO8 & 8.28 & 27.50 & 0.00 & 23.48 & 0.00 & 23.48 \\
\hline Cost [€] & 1876.40 & 655.09 & \multicolumn{2}{|c|}{655.09} \\
\hline
\end{tabular}

TABLE VI

THE GBFS IN CASE OF X13-X14-X15 CONGESTION

\begin{tabular}{|c|c|c|c|c|c|c|}
\hline \multirow{2}{*}{ Network } & \multicolumn{2}{|c|}{$j=1$} & \multicolumn{2}{c|}{$j=2$} & \multicolumn{2}{c|}{$j=3$} \\
\cline { 2 - 7 } & $P^{\text {sum }}$ & $\alpha$ & $P^{\text {sum }}$ & $\alpha$ & $P^{\text {sum }}$ & $\alpha$ \\
\hline DNO & 20.03 & 8.00 & 52.65 & 18.00 & 52.65 & 18.00 \\
LNO1 & 7.08 & 24.00 & 0.00 & 20.00 & 0.00 & 20.00 \\
LNO2 & 6.53 & 24.50 & 0.00 & 20.50 & 0.00 & 20.50 \\
LNO3 & 9.97 & 26.50 & 0.00 & 21.00 & 0.00 & 21.00 \\
LNO4 & 10.47 & 27.00 & 0.00 & 21.50 & 0.00 & 21.50 \\
LNO5 & 14.58 & 28.00 & 5.86 & 24.00 & 5.86 & 24.00 \\
LNO6 & 10.68 & 28.00 & 0.00 & 22.50 & 0.00 & 22.49 \\
LNO7 & 6.14 & 27.00 & 0.00 & 23.00 & 0.00 & 22.99 \\
LNO8 & 8.27 & 27.50 & 0.00 & 23.50 & 0.00 & 23.49 \\
\hline Cost [€] & 1888.70 & 662.38 & \multicolumn{2}{|c|}{662.38} \\
\hline
\end{tabular}

accurate results than DCOPF. The tightness of the relaxation from the power loss constraints in the SOC-ACOPF can be guaranteed by the proposed sequential tightness algorithm. The issue of network information sharing is addressed through the proposed concept of GBF. The HED mechanism is also proposed to decompose the dispatch task of multiple connected networks. A three-level network is considered. At the third level, LNOs communicate the dispatch cost of their networks through the proposed GBFs. At the second level, DNO runs another optimization considering its own network conditions 
TABLE VII

THE GBFS IN CASE OF X16-X17-X18 CONGESTION

\begin{tabular}{|c|c|c|c|c|c|c|}
\hline \multirow{2}{*}{ Network } & \multicolumn{2}{|c|}{$j=1$} & \multicolumn{2}{c|}{$j=2$} & \multicolumn{2}{c|}{$j=3$} \\
\cline { 2 - 7 } & $P^{\text {sum }}$ & $\alpha$ & $P^{\text {sum }}$ & $\alpha$ & $P^{\text {sum }}$ & $\alpha$ \\
\hline DNO & 20.03 & 8.00 & 52.60 & 18.00 & 0.00 & 18.00 \\
LNO1 & 7.08 & 24.00 & 0.00 & 20.00 & 0.00 & 20.00 \\
LNO2 & 6.53 & 24.50 & 0.00 & 20.50 & 0.00 & 20.50 \\
LNO3 & 9.97 & 26.50 & 0.00 & 21.00 & 0.00 & 21.00 \\
LNO4 & 10.47 & 27.00 & 0.00 & 21.50 & 0.00 & 21.49 \\
LNO5 & 10.66 & 27.50 & 0.00 & 22.00 & 5.97 & 21.99 \\
LNO6 & 14.66 & 28.50 & 5.97 & 24.50 & 0.00 & 24.50 \\
LNO7 & 6.14 & 27.00 & 0.00 & 23.00 & 0.00 & 22.99 \\
LNO8 & 8.27 & 27.50 & 0.00 & 23.50 & 0.00 & 23.49 \\
\hline Cost [€] & 1892.15 & \multicolumn{6}{|c|}{666.96} & \multicolumn{2}{c|}{666.96} \\
\hline
\end{tabular}

TABLE VIII

THE GBFS IN CASE OF X21-X22 CONGESTION

\begin{tabular}{|c|c|c|c|c|c|c|}
\hline \multirow{2}{*}{ Network } & \multicolumn{2}{|c|}{$j=1$} & \multicolumn{2}{|c|}{$j=2$} & \multicolumn{2}{|c|}{$j=3$} \\
\hline & $P^{\text {sum }}$ & $\alpha$ & $P^{\text {sum }}$ & $\alpha$ & $P^{\text {sum }}$ & $\alpha$ \\
\hline DNO & 20.00 & 8.00 & 52.54 & 18.00 & 52.54 & 18.00 \\
\hline LNO1 & 7.08 & 24.00 & 0.00 & 19.96 & 0.00 & 20.00 \\
\hline LNO2 & 6.53 & 24.50 & 0.00 & 20.46 & 0.00 & 20.49 \\
\hline LNO3 & 9.96 & 26.50 & 0.00 & 20.97 & 0.00 & 20.99 \\
\hline LNO4 & 10.47 & 27.00 & 0.00 & 21.47 & 0.00 & 21.49 \\
\hline LNO5 & 10.65 & 27.50 & 0.00 & 21.94 & 0.00 & 21.99 \\
\hline LNO6 & 10.67 & 28.00 & 0.00 & 22.44 & 0.00 & 22.49 \\
\hline LNO7 & 6.14 & 27.00 & 0.00 & 22.95 & 0.00 & 22.99 \\
\hline LNO8 & 12.45 & 31.50 & 6.13 & 27.50 & 6.13 & 27.50 \\
\hline Cost $[€]$ & \multicolumn{2}{|c|}{1900.90} & \multicolumn{2}{|c|}{$\frac{1}{676.38}$} & \multicolumn{2}{|c|}{676.38} \\
\hline
\end{tabular}

and submitted GBFs of the LNOs. The results of this optimization are packed in the form of GBFs and communicated to the first level of hierarchy i.e. TNO. Once the optimization problem of the first level of hierarchy is solved, the dispatch results and nodal prices are communicated back down to the hierarchy. The convergence of HED mechanism is guaranteed by the convexity of the SOC-ACOPF. The simulation results show the utility of proposed mechanism for implementing nodal pricing in distribution and local networks. The proposed mechanism is tested in a stationary environment. Studying the impact of uncertainty is a possible future extension of this work.

\section{ACKNOWLEDGMENT}

The authors would like to thank the support from SETS Erasmus Mundus Joint Doctorate Fellowship for this research project on distribution pricing. We want to express our special thanks to Konstantin Staschus who is the Secretary-General of the European Network of Transmission System Operators (ENTSO-E). Dr. Konstantin Staschus provides many insightful and highly motivating comments on this paper when the first draft is ready. These comments help the authors to improve the paper before the final submission to IEEE. All the careful reviews on this paper by the editors and reviewers of this journal are sincerely appreciated by the authors.

\section{REFERENCES}

[1] P. M. Sotkiewicz and J. M. Vignolo, "Nodal pricing for distribution networks: efficient pricing for efficiency enhancing DG," Power Systems, IEEE Transactions on, vol. 21, no. 2, pp. 1013-1014, 2006.

[2] R. Li, Q. Wu, and S. Oren, "Distribution locational marginal pricing for optimal electric vehicle charging management," Power Systems, IEEE Transactions on, vol. 29, no. 1, pp. 203-211, 2014.
[3] S. Widergren, C. Marinovici, T. Berliner, and A. Graves, "Real-time pricing demand response in operations," in Power and Energy Society General Meeting, 2012 IEEE, 2012, pp. 1-5.

[4] F. Sahriatzadeh, P. Nirbhavane, and A. Srivastava, "Locational marginal price for distribution system considering demand response," in North American Power Symposium (NAPS), 2012, 2012, pp. 1-5.

[5] S. Huang, Q. Wu, S. S. Oren, R. Li, and Z. Liu, "Distribution locational marginal pricing through quadratic programming for congestion management in distribution networks," IEEE Transactions on Power Systems, vol. 30, no. 4, pp. 2170-2178, 2015.

[6] G. T. Heydt, B. H. Chowdhury, M. L. Crow, D. Haughton, B. D. Kiefer, F. Meng, and B. R. Sathyanarayana, "Pricing and control in the next generation power distribution system," Smart Grid, IEEE Transactions on, vol. 3, no. 2, pp. 907-914, 2012.

[7] R. Mudumbai and S. Dasgupta, "Distributed control for the smart grid: The case of economic dispatch," in Information Theory and Applications Workshop (ITA), 2014, 2014, pp. 1-6.

[8] H. Karami, M. Sanjari, S. Hosseinian, and G. Gharehpetian, "An optimal dispatch algorithm for managing residential distributed energy resources," IEEE Transactions on Smart Grid, vol. 5, no. 5, pp. 23602367, 2014.

[9] A. Fazeli, M. Sumner, C. Johnson, and E. Christopher, "Coordinated optimal dispatch of distributed energy resources within a smart energy community cell," in Innovative Smart Grid Technologies (ISGT Europe), 2012 3rd IEEE PES International Conference and Exhibition on, 2012, pp. $1-10$.

[10] A. J. Conejo and J. A. Aguado, "Multi-area coordinated decentralized DC optimal power flow," IEEE Transactions on Power Systems, vol. 13, no. 4, pp. 1272-1278, 1998.

[11] Y. Zhang and F. Li, "Network pricing for high voltage radial distribution networks," in Power and Energy Society General Meeting, 2011 IEEE, 2011, pp. 1-5.

[12] V. Loia and A. Vaccaro, "Decentralized economic dispatch in smart grids by self-organizing dynamic agents," IEEE Transactions on Systems, Man, and Cybernetics: Systems, vol. 44, no. 4, pp. 397-408, 2014.

[13] F. Meng and B. Chowdhury, "Distribution LMP-based economic operation for future smart grid," in Power and Energy Conference at Illinois (PECI), 2011 IEEE, 2011, pp. 1-5.

[14] P. Hallberg et al., "Active distribution system management a key tool for the smooth integration of distributed generation," Eurelectric TF Active System Management, vol. 2, p. 013, 2013.

[15] E. Rivero, S. Daan, A. Ramos, M. Maenhoudt, and A. Ulian, "Preliminary assessment of the future role of DSOs, future market architectures and regulatory frameworks for network integration of dres," European Project EvolvDSO, Deliverable, vol. 1, 2014.

[16] K. Purchala, L. Meeus, D. Van Dommelen, and R. Belmans, "Usefulness of DC power flow for active power flow analysis," in IEEE Power Engineering Society General Meeting, $2005 . \quad$ IEEE, 2005, pp. 454459.

[17] M. Farivar and S. H. Low, "Branch flow model: Relaxations and convexification Part I," IEEE Transactions on Power Systems, vol. 28, no. 3, pp. 2554-2564, 2013.

[18] L. Gan, N. Li, U. Topcu, and S. H. Low, "Exact convex relaxation of optimal power flow in radial networks," Automatic Control, IEEE Transactions on, vol. 60, no. 1, pp. 72-87, 2015.

[19] M. Baradar and M. R. Hesamzadeh, "AC power flow representation in conic format," IEEE Transactions on Power Systems, vol. 30, no. 1, pp. 546-547, 2015.

[20] X. Bai, H. Wei, K. Fujisawa, and Y. Wang, "Semidefinite programming for optimal power flow problems," International Journal of Electrical Power \& Energy Systems, vol. 30, no. 6, pp. 383-392, 2008.

[21] J. Lavaei and S. H. Low, "Zero duality gap in optimal power flow problem," Power Systems, IEEE Transactions on, vol. 27, no. 1, pp. 92-107, 2012.

[22] R. Madani, M. Ashraphijuo, and J. Lavaei, "Promises of conic relaxation for contingency-constrained optimal power flow problem," IEEE Transactions on Power Systems, vol. 31, no. 2, pp. 1297-1307, March 2016.

[23] B. C. Lesieutre, D. K. Molzahn, A. R. Borden, and C. L. DeMarco, "Examining the limits of the application of semidefinite programming to power flow problems," in Communication, Control, and Computing (Allerton), 2011 49th Annual Allerton Conference on. IEEE, 2011, pp. 1492-1499.

[24] J. Lavaei, D. Tse, and B. Zhang, "Geometry of power flows and optimization in distribution networks," Power Systems, IEEE Transactions on, vol. 29, no. 2, pp. 572-583, 2014. 
[25] S. Low, "Convex relaxation of optimal power flow, part i: Formulations and equivalence," Control of Network Systems, IEEE Transactions on, vol. 1, no. 1, pp. 15-27, March 2014.

[26] - "Convex relaxation of optimal power flow, part ii: Exactness," Control of Network Systems, IEEE Transactions on, no. 2, pp. 177-189, June 2014.

[27] B. Kocuk, S. Dey, and X. Sun, "Inexactness of sdp relaxation and valid inequalities for optimal power flow," Power Systems, IEEE Transactions on, to be published, early Access. [Online]. Available: http://ieeexplore.iee.. rg/stamp/stamp.jsp?arnumber=7056568

[28] R. Jabr et al., "Radial distribution load flow using conic programming," Power Systems, IEEE Transactions on, vol. 21, no. 3, pp. 1458-1459, 2006.

[29] X. Kuang, L. F. Zuluaga, B. Ghaddar, and J. Naoum-Sawaya, "Approximating the acopf problem with a hierarchy of socp problems," in 2015 IEEE Power Energy Society General Meeting, July 2015, pp. 1-5.

[30] D. K. Molzahn and I. A. Hiskens, "Mixed sdp/socp moment relaxations of the optimal power flow problem," in PowerTech, 2015 IEEE Eindhoven, June 2015, pp. 1-6.

[31] S. Bose, S. H. Low, T. Teeraratkul, and B. Hassibi, "Equivalent relaxations of optimal power flow," IEEE Transactions on Automatic Control, vol. 60, no. 3, pp. 729-742, March 2015.

[32] B. Kocuk, S. S. Dey, and X. A. Sun, "Strong socp relaxations for the optimal power flow problem," Operations Research, 2016.

[33] A. Mosek, "The MOSEK optimization software," Online at http://www. mosek. com, vol. 54, 2010.

[34] K. Lehmann, A. Grastien, and P. V. Hentenryck, "AC-Feasibility on Tree Networks is NP-Hard," IEEE Transactions on Power Systems, vol. 31, no. 1, pp. 798-801, Jan 2016.

[35] A. Verma, "Power grid security analysis: an optimization approach," Ph.D. dissertation, Dept. Ind. Eng. Oper. Res., Columbia Univ., New York, NY, USA, 2009.

[36] A. V. Fiacco and J. Kyparisis, "Convexity and concavity properties of the optimal value function in parametric nonlinear programming," Journal of Optimization Theory and Applications, vol. 48, no. 1, pp. 95-126, 1986.

[37] A. J. Conejo, E. Castillo, R. Minguez, and R. Garcia-Bertrand, Decomposition techniques in mathematical programming: engineering and science applications. Springer Science \& Business Media, 2006.

[38] K. Schneider, P. Phanivong, and J.-S. Lacroix, "IEEE 342-node low voltage networked test system," in PES General Meeting-Conference \& Exposition, 2014 IEEE. IEEE, 2014, pp. 1-5.

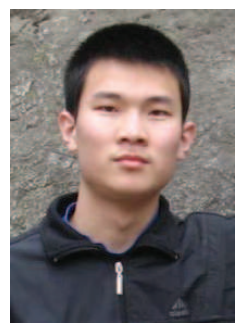

Zhao Yuan received B.E. in Electrical Engineering from Hebei University of Technology in 2011, double M.E. in Electrical Engineering from Huazhong University of Science and Technology and in Renewable Energy from ParisTech in 2014. He is currently pursuing the $\mathrm{PhD}$ degree in Electricity Market Research Group (EMReG) of KTH Royal Institute of Technology, Sweden. His research interests include optimal power flow, power system operation and electricity market.

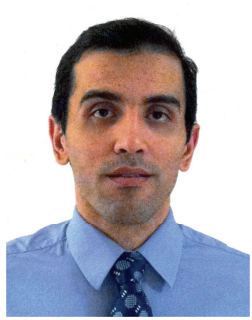

Mohammad Reza Hesamzadeh (IEEE-SM13) received his Docent from KTH Royal Institute of Technology, Sweden, and his PhD from Swinburne University of Technology, Australia, in 2013 and 2010 respectively. He was a post-doctoral fellow at KTH in 2010-2011 where he is currently a faculty member. His Special fields of interests include Electricity market modeling, analysis, and design and mathematical modelling and computing. Dr Hesamzadeh is a member of International Association for Energy Economics (IAEE) and a Member

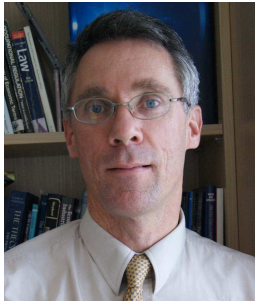

Darryl R. Biggar is an economist with the Australian Competition and Consumer Commission and the Australian Energy Regulator. He specializes in the economics of regulation, including issues such as the design of incentive mechanisms, foundations of regulation, and the design of electricity markets and water markets. He has a particular interest in electricity markets including issues of nodal and zonal pricing and the measurement and control of market power. Prior to the ACCC, he worked for the OECD in Paris, the New Zealand government, and for University College, London. Biggar has a $\mathrm{PhD}$ in economics from Stanford University and an MA in Mathematics from Cambridge University. $\mathrm{He}$ is a native of New Zealand.

of Cigre, Sweden Section. 